\title{
Effect of D-penicillamine treatment on brain metabolism in Wilson's disease: a case study
}

\author{
A DE VOLDER, C J M SINDIC,* A M GOFFINET \\ From the Positron Tomography Laboratory, University of Louvain, Louvain-la-Neuve, and the Neurology \\ Service,* Cliniques Universitaires St Luc, Brussels, Belgium
}

SUMMARY Sequential measurements of brain glucose metabolism were carried out in a patient with Wilson's disease, before and after successful treatment with D-penicillamine. They demonstrate an evolution of regional metabolism consistent with clinical improvement. The first study showed marked hypometabolism in the putamen on both sides. The second analysis showed bilateral improvement, with predominant residual deficits in the right putamen, while clinical symptoms of striatal dysfunction persisted on the left side. This observation suggests that positron emission tomography is able to follow the neurological evolution in cases of Wilson's disease.

Wilson's disease (WD), or hepatolenticular degeneration ${ }^{1}$ is mainly characterised by copper deposits in liver, brain and cornea. ${ }^{2}$ Among the treatments proposed, the copper chelator D-penicillamine is generally favoured. ${ }^{34}$ Other molecules such as triethylene tetramine dihydrochloride ${ }^{5}$ or zinc sulphate $^{78}$ are usually reserved for those cases where intolerance to D-penicillamine occurs. ${ }^{9}$ In the brain, the main targets of the disease are the putamen and the pallidum; caudate nuclei and other brain structures such as cerebellar dentate nuclei are also affected. ${ }^{10-12}$ Striatal dysfunction is generally held responsible for the cardinal neurological symptoms of dystonia, axial tremor and other extrapyramidal signs. Previous papers ${ }^{13}{ }^{14}$ have reported a diffuse decrease of brain glucose utilisation associated with relative focal hypometabolism in the lenticular nuclei of four patients with Wilson's disease. Three of these patients received penicillamine but no pretreatment measurements were obtained so that the effect of treatment on metabolic parameters has not been studied so far.

The present work describes two studies of glucose metabolism performed in the same patient before and after 17 months of D-penicillamine treatment. The results suggest a strong correlation between regional brain metabolism and response to therapy in Wilson's disease.

\section{Address for reprint requests: Anne G De Volder, Positron Tomog- raphy Laboratory, 2, Chemin du Cyclotron, B-1348 Louvain-la- Neuve, Belgium.}

Received 21 November 1987 and in revised form 16 February 1988. Accepted 1 March 1988

\section{Patient history}

The propositus, a white Caucasian female, was aged 20 years at the time of diagnosis and of the first study. She was hospitalised because of the appearance of a very marked tremor of head and hands during the last few months. Writing was impossible and dystonia of the trunk was present when walking. Head tremor was typically blocked with both hands by the patient herself. Clinical examination disclosed bilateral Kayser-Fleischer rings. Previous medical problems consisted of haemolytic anaemia (dating back 7 years) and primary amenorrhoea; these were early manifestations of the disease. The diagnosis of Wilson's disease was confirmed by the very low levels of serum coeruloplasmin $(6 \mathrm{mg} / 100 \mathrm{ml}$; reference values: $29-36)$ and copper $31 \mu \mathrm{g} / 100 \mathrm{ml}$ (reference values: 83-153). Laparoscopy revealed the presence of a macronodular cirrhosis and splenomegaly. The copper content in the liver was remarkably high $(750 \mu \mathrm{g} / \mathrm{g}$ dry weight). Serum liver enzymes were in the normal range. Discrete oesophageal varicosities were observed by endoscopy. D-penicillamine treatment was started and the dose increased up to $1.5 \mathrm{~g} /$ day. The tremor disappeared almost completely within 12 months and spontaneous menstruation occurred for the first time after 18 months of treatment. Some dystonia and functional impairment persisted in the left hand.

\section{Methods \\ Regional brain glucose metabolism was measured using pos- itron emission tomography (PET) with [F-18]2 deoxy-2- fluoro-D-glucose (FDG), according to the method of Phelps et al. ${ }^{15}$ Measurements were made with an ECAT III tomog- raph (CTI) with an in-plane resolution of $9 \mathrm{~mm}$ FWHM and a slice thickness of $15 \mathrm{~mm} .{ }^{16}$ FDG was injected intra- venously, and the input function was sampled from a radial artery. Eight slices parallel to the canthomeatal line were}


Table 1 Metabolic values. (rCMRGLc, expressed in umol/100 $\mathrm{g} \mathrm{min)}$

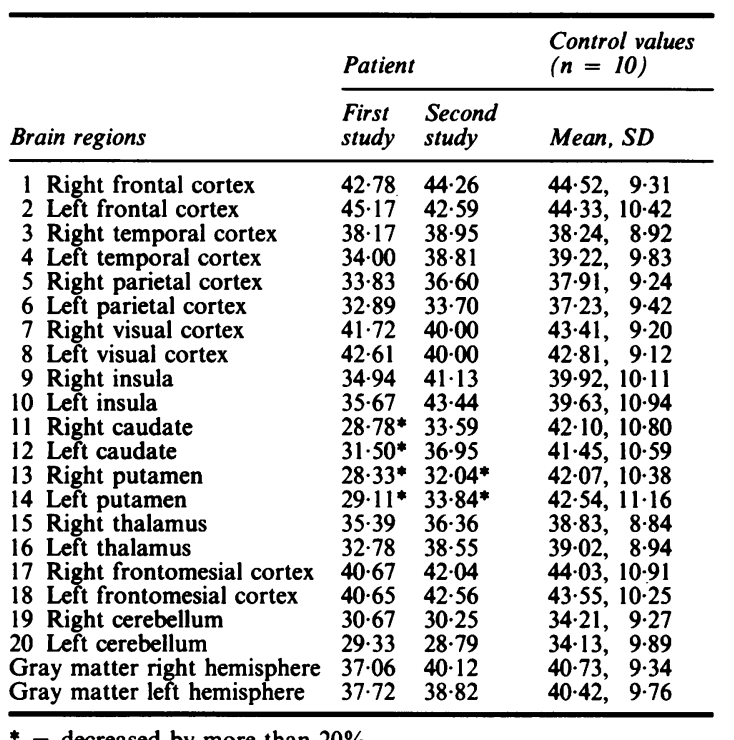

* = decreased by more than $20 \%$.

collected at $1 \mathrm{~cm}$ intervals, starting from the level of the cerebellum. The overlap between adjacent sections ensured a complete sampling of the activity contained in basal nuclei. Glucose metabolic rates were calculated using the following set of rate constants: $\mathbf{k} 1=0.092 ; \mathbf{k} 2=0.140 ; \mathbf{k} 3=0.075$; k4 $=0.0056$; lumped constant $=0.420$. Control studies were carried out in 10 normal, age-matched volunteers. Regions of interest were drawn on caudate nuclei and putamina by reference to neuroanatomical landmarks, particularly the thalamus which was prominent on PET images, and to CT scans. Absolute metabolic rates were considered abnormal when deviating by more than $20 \%$ from control values. Relative metabolic rates were expressed in percent of mean gray metabolism. They were considered abnormal when differing from corresponding values in control subjects by more than 2 standard deviations.

\section{Results and discussion}

Absolute and relative metabolic rates in the propositus and in control subjects are shown in tables 1 and 2. A perfect match in the head's position between the two studies could not be achieved. However, the data collection mode was such that the entire volume of basal nuclei was sampled in adjacent, slightly overlapping sections. Regions of interest were defined on the head of the caudate nuclei and on putamina in the images where these structures were best visible (that is, in the second study). They were transferred and matched on the corresponding image of the first analysis as shown in the fig. It is readily apparent that mean gray glucose metabolism was affected neither by the disease nor by the treatment. In the first study (fig a), the putamen and, to a lesser extent, the caudate nuclei were the only regions with significant hypometabolism. After 17 months of treatment (fig b), glucose utilisation remained normal in all brain regions which were normal in the first study. There was $10 \%$ improvement of both relative and absolute metabolic rates in the putamen on both sides, however with a slightly larger deficit on the right side. After treatment, the putaminal anomalies were borderline in terms of absolute metabolic rates, but still clearly significant in terms of relative rates. In the caudate nuclei, post-treatment metabolic values were within the normal range.

In the present patient, mean brain glucose metabolism was not affected by Wilson's disease. This is in contrast to the study of Hawkins et al ${ }^{13}$ who found diffuse glucose hypometabolism in four Wilson's disease patients. It should be pointed out, however, that these patients had a long history of disease and apparently severe neurological and hepatic changes.

Our measurements suggest that, with the exception of the putamen and caudate nuclei, brain glucose metabolism is not affected by Wilson's disease, at least during the first stages of the disease. In spite of its common pathological involvement, the cerebellum, in particular, did not show any significant anomaly in the present study. As might be predicted, Dpenicillamine treatment did not alter global brain metabolism and resulted in a remarkable but incomplete recovery of glucose utilisation in the striatum. The metabolic and clinical evolutions were correlated: the right putamen remained, after treatment, slightly more affected than its left counterpart. This is in

Table 2 Relative values. (rCMRGLc/Mean CMRGLc, expressed in \%)

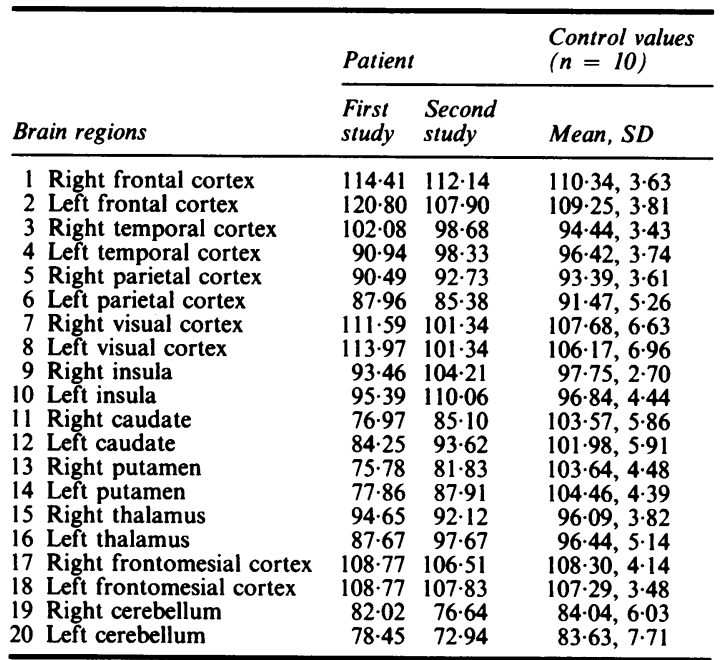




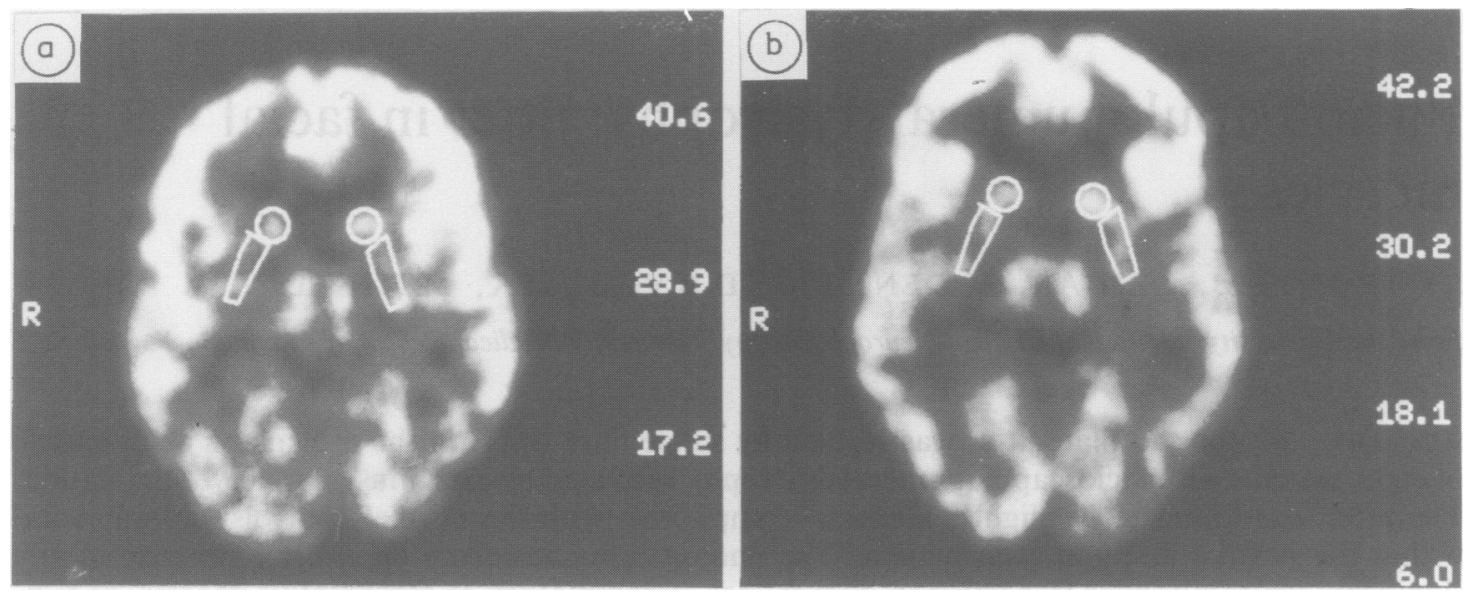

Figure PET images at the level of the basal nuclei, before $(A)$, and after $(B)$ treatment by D-penicillamine. Prominent hypometabolism was found in both putamina in the first PET examination (A), whilst the second study (B) showed a marked but incomplete improvement of glucose utilisation in the putamen, especially on the left side. Regions of interest have been drawn on caudate nuclei and putamina.

agreement with the persisting dystonia on the left side. PET, however, demonstrated the persistence of metabolic anomalies on both sides, despite the satisfactory clinical evolution, and may thus provide a more sensitive estimation of the neurological involvement than does clinical assessment. Owing to the large individual variations of global glucose utilisation rates, the estimation of relative metabolic indexes probably gives sufficient information, thus eliminating the need of an arterial input function. Provided this impression is confirmed in further cases, our observation suggests that the PET/FDG method may be a suitable tool for following the neurological evolution of Wilson's disease.

We thank Dr F De Ridder for bringing this patient to our attention and the staff of the Positron Tomography Laboratory for assistance with the PET measurements. ADV is research assistant and AG is senior research associate at the FNRS. This work was supported by FRSM grants 3.4540.81 and 3.4540.84.

\section{References}

1 Wilson SAK. Progressive lenticular degeneration: a familiar nervous disease associated with cirrhosis of the liver. Brain 1912;34:295-509.

2 Walshe JM. Wilson's disease, a review. In: Peisach J, Aisen P, Blumberg WE, eds. The Biochemistry of Copper. New York: Academic Press, 1966:475-98.

3 Walshe JM. Penicillamine, a new oral therapy for Wilson's disease. Am J Med 1956;21:487-95.

4 Sternlieb I, Scheinberg IH. Prevention of Wilson's disease in asymptomatic patients. $N$ Engl J Med 1968; 278:352-9.

5 Walshe JM. Treatment of Wilson's disease with Trientine (triethylene tertramine) dihydrochloride. Lancet 1982;i:643-7.
6 Scheinberg IH, Jaffe ME, Sternlieb I. The use of Trientine in preventing the effects of interrupting Penicillamine therapy in Wilson's disease. $N$ Engl J Med 1987;317:209-13.

7 Brewer GJ, Hill GM, Prasad AS, Cossack ZT, Rabbani P. Oral Zinc therapy for Wilson's disease. Ann Intern Med 1983;99:314-20.

8 Hoogenraad TU, Van Hattum J, Van den Hamer CJA. Management of Wilson's disease with zinc sulphate. Experience in a series of 27 patients. $J$ Neurol Sci 1987;77:137-46.

9 Deiss A. Treatment of Wilson's disease. Ann Intern Med 1983;99:398-400.

10 Walshe JM. Wilson's disease (hepatolenticular degeneration). In: Vinken PJ, Bruyn GW, Klawans HL, eds. Handbook of Clinical Neurology, Vol 27. Amsterdam: North-Holland, 1976:379-414.

11 Smith WT. Wilson's disease. In: Blackwood W, Corsellis JAN, eds. Greenfield's Neuropathology. London: Edward Arnold Publ, 1976:172-7.

12 Schulman S. Wilson's disease. In: Minckler J, ed. Pathology of the Nervous System, Vol 1. New York: McGraw Hill, 1968:1139-52.

13 Hawkins RA, Mazziotta JC, Phelps ME. Wilson's disease studied with FDG and PET. Neurology 1987;37:1707-11.

14 Mazziotta JC, Phelps ME. Positron Emission tomography studies of the Brain. In: Phelps ME, Mazziotta JC, Schelbert HR, eds. Positron Emission Tomography and Autoradiography: Principles and Applications for the Brain and Heart. New York: Raven Press, 1986; 563-4.

15 Phelps ME, Huang SC, Hoffman EJ, Selin C, Sokoloff L, Kuhl DE. Tomographic measurement of local cerebral glucose metabolic rate in humans with [F-18]2-Fluoro2-Deoxy-D-Glucose: Validation of Method. Ann Neurol 1979;6:371-88.

16 Hoffman EJ, Phelps ME, Huang SC, et al. Dynamic, gated and high resolution imaging with the ECAT III. IEEE Trans Nucl Sci 1986;33:452-5. 